\title{
The Herschel view of the Galactic center
}

\author{
John Bally and the Hi-GAL team \\ Center for Astrophysics and Space Astronomy, \\ Department of Astrophysical and Planetary Sciences \\ University of Colorado at Boulder, \\ UCB 389, Boulder, Colorado, USA \\ email: john.bally@colorado.edu
}

\begin{abstract}
The 3.5 meter diameter Herschel Space Observatory conducted a 720 square-degree survey of the Galactic plane, the Herschel Galactic plane survey (Hi-GAL). These data provide the most sensitive and highest resolution observations of the far-IR to sub-mm continuum from the central molecular zone (CMZ) at $\lambda=70,160,250,350$, and $500 \mu \mathrm{m}$ obtained to date. $\mathrm{Hi}-\mathrm{GAL}$ can be used to map the distributions of temperature and column density of dust in CMZ clouds, warm dust in HiI regions, and identify highly embedded massive protostars and clusters and the dusty shells ejected by supergiant stars. These data enable classification of sources and re-evaluation of the current and recent star-formation rate in the CMZ. The outer CMZ beyond $|l|=0.9$ degrees $\left(R_{\text {gal }}>130 \mathrm{pc}\right)$ contains most of the dense $\left(n>10^{4} \mathrm{~cm}^{-3}\right)$ gas in the Galaxy but supports very little star formation. The Hi-GAL and Spitzer data show that almost all star formation occurs in clouds moving on $x_{2}$ orbits at $R_{\text {gal }}<100 \mathrm{pc}$. While the $10^{6}$ $\mathrm{M}_{\odot}$ Sgr B2 complex, the $50 \mathrm{~km} \mathrm{~s}^{-1}$ cloud near Sgr A, and the $\operatorname{Sgr} \mathrm{C}$ region are forming clusters of massive stars, other clouds are relatively inactive star formers, despite their high densities, large masses, and compact sizes. The asymmetric distribution of dense gas about Sgr $\mathrm{A}^{*}$ on degree scales (most dense CMZ gas and dust is at positive Galactic longitudes and positive $V_{L S R}$ ) and compact $24 \mu \mathrm{m}$ sources (most are at negative longitudes) may indicate that eposidic mini-starbursts occasionally 'blow-out' a portion of the gas on these $x_{2}$ orbits. The resulting massive-star feedback may fuel the compact $30 \mathrm{pc}$ scale Galactic center bubble associated with the Arches and Quintuplet clusters, the several hundred pc scale Sofue-Handa lobe, and the kpc-scale Fermi/LAT bubble, making it the largest 'superbubble' in the Galaxy. A consequence of this model is that in our Galaxy, instead of the supermassive black hole (SMBH) limiting star formation, star formation may limit the growth of the SMBH.
\end{abstract}

\section{Introduction}

In this first talk at IAU 303, I will give an overview of the central molecular zone (CMZ), introduce some recent results from the Herschel Galactic plane survey (Hi-GAL) as they pertain to the CMZ, and discuss some outstanding problems in the investigation of the Galactic center and its immediate environment.

The Milky Way is a mildly barred galaxy. Analyses of the kinematics of CO and HI emission from the central $3 \mathrm{kpc}$, in particular the behavior of the radial velocity of the gas as a function of Galactic longitude, led to the conclusion that gas motions in the inner Galaxy are dominated by a stellar bar extending to a galactocentric radius of about $3 \mathrm{kpc}$ (Binney et al. 1991; Morris \& Serabyn 1996). The bar's major axis is inclined with respect to our line-of-sight by about $20^{\circ}$ to $45^{\circ}$ with the closer part being in the first quadrant (Figure 1). The shape of the near-infrared light from the Galactic bulge confirms this interpretation (Blitz et al. 1993). The azimuthally averaged surface density of interstellar gas reaches a peak around 3 to $4 \mathrm{kpc}$, dips by nearly an order of magnitude between 0.5 to $3 \mathrm{kpc}$, the region dominated by the stellar bar. 


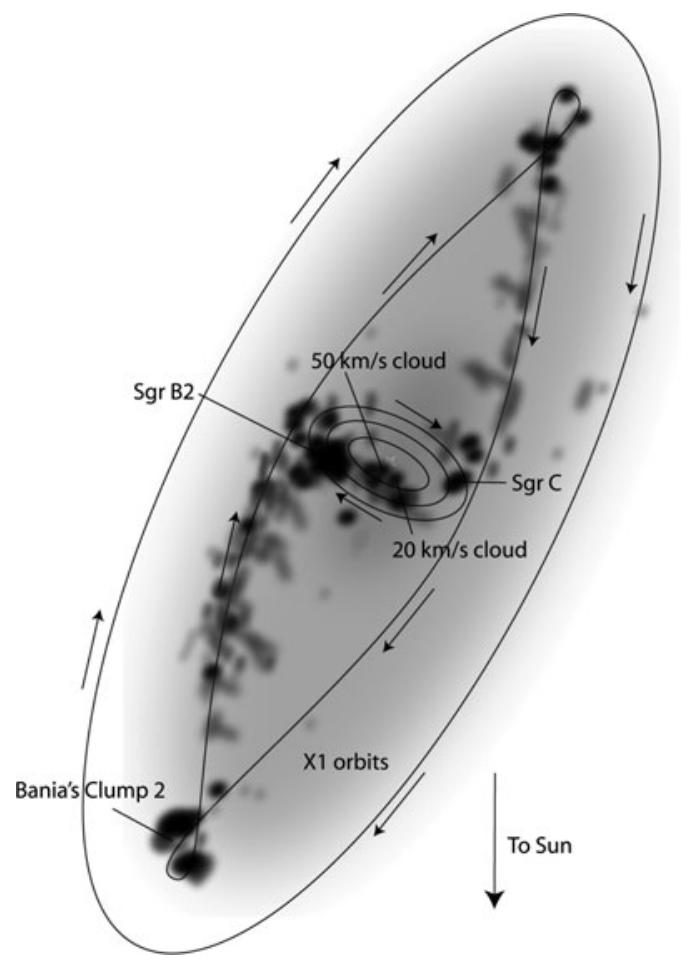

Figure 1. A cartoon showing a face-on view of the central $500 \mathrm{pc}$ region of the Milky Way as viewed from the northern Galactic pole. The Sun is located below the figure and positive longitudes to the left. The diffuse grey-scale shows the current orientation of the stellar bar, thought to have its major axis tilted between 20 to 45 degrees with respect to our line-of sight. The large oval shows a non intersecting $x_{1}$ orbit; lower angular momentum $x_{1}$ orbits become self-intersecting. The "rhombus" of molecular emission in $l-V$ diagrams probably occupies the receding and approaching portions of the innermost $x_{1}$ orbits. The 3 smaller inscribed ellipses show $x_{2}$ orbits. Approaching gas presumably enters as an atomic or low-density molecular medium; shock-compression and subsequent cooling results in its conversion to the dense molecular phase. Bania's Clump 2 could be located at the location of the inner-most self-intersecting $x_{1}$ orbits as shown, represent gas along the leading edge of the bar, or where this dust lane intersects the outer $x_{2}$ orbits. The CMZ emission is associated with gas mostly located on the near-side of the Galactic center on the $x_{2}$ orbits. The possible locations of the Sgr C, $20 \mathrm{~km} \mathrm{~s}^{-1}$ cloud, $50 \mathrm{~km} \mathrm{~s}^{-1}$ cloud, and Sgr B2 complexes are indicated.

The CMZ, which occupies the central 500 pc, contains 5 to $10 \%$ of the molecular gas mass of the Galaxy, hosts the largest concentration of massive stars and star clusters, (Morris \& Serabyn 1996, Ferriere, Gillard, \& Jean 2007) and two of the most massive, densest, and luminous star-forming complexes in the Milky Way, Sgr A and Sgr B2 (YusefZadeh et al. 2008; 2009). Giant molecular clouds (GMCs) in the CMZ are one to two orders of magnitude denser and have over an order of magnitude larger line-widths than GMCs in the Galactic plane beyond $3 \mathrm{kpc}$ (Bally et al. 1987; 1988; Oka et al. 1998; Tsuboi et al. 1999; Oka et al. 2001). The CMZ and the immediate vicinity of the central black hole allows the study of star and cluster formation in extreme environments, bridging the link between low-rates of star birth in the Solar vicinity and high-rates in starburst galaxies. The CMZ may be similar to the star-forming rings observed in the centers of some galaxies such as NGC 1097 (Kormendy \& Kennicutt 2004). Unfortunately, our vantage point makes it very difficult the determine the true geometry and distribution of the CMZ gas, dust, and stellar populations. 
The CMZ contains $\sim 3-7 \times 10^{7} \mathrm{M}_{\odot}$ of high-density $\left(<n\left(H_{2}\right)>>10^{4} \mathrm{~cm}^{-3}\right)$ molecular gas (Bally et al. 1987, 1988; Morris, \& Serabyn, 1996; Ferriere, Gillard, \& Jean 2007; Longmore et al. 2013a,b). Do massive stars and star clusters dominate feedback, regulate the state and energetics of the interstellar medium (ISM), and the rate at which the ISM is converted into stars? Their ionizing radiation, stellar winds, and supernovae power superbubbles can reach many kilo-parsecs above and below the Galactic plane. What role, if any, does the $3.6 \times 10^{6} \mathrm{M}_{\odot}$ supermassive black hole $(\mathrm{SMBH})$ play in regulating the state of the CMZ? The CMZ is the only galactic nucleus sufficiently close for the study of these physical processes on milli-parsec scales required to resolve individual stars and star-forming cores.

The CMZ exhibits diffuse and variable X-ray emission in the $6.4 \mathrm{keV}$ fluorescent $\mathrm{K}-\alpha$ line produced by neutral iron atoms (Clavel et al. 2013). The accepted interpretation is that $\mathrm{Sgr} \mathrm{A}^{*}$ has experienced major outbursts within the last few hundred years. Although the current X-ray luminosity of Sgr A* hovers around $\mathrm{L}_{x} \sim 10^{34} \mathrm{erg} \mathrm{s}^{-1}$ within a factor of a few (the X-ray emission is variable on a time-scale of hours to days), the 6.4 $\mathrm{keV}$ emission indicates past flares with $\mathrm{L}_{x} \sim 10^{39} \mathrm{erg} \mathrm{s}^{-1}$. As the resulting X-ray flash propagates through the $\mathrm{CMZ}$, it produces variable $6.4 \mathrm{keV}$ emission which is effectively an X-ray light echo.

The Galactic center powers a bipolar outflow whose outer boundaries reach $\sim 6 \mathrm{kpc}$ above and below the Galactic plane. First detected at centimeter wavelength by the WMAP satellite as the 'WMAP haze', the bipolar bubbles were confirmed by the Planck satellite (Planck Collaboration: Ade et al. 2013), and ground-based measurements of polarized, centimeter wavelength radio continuum emission (Carretti et al. 2013). Energetic $\gamma$-rays with $\mathrm{GeV}$ energies have been detected from the interior of this feature by the LAT detector on the Fermi satellite (the 'Fermi/LAT bubble'; Su, Slatyer, \& Finkbeiner 2010).

Was the kpc-scale Fermi/LAT bubble energized by past outburst activity of the SMBH? If so, the Fermi bubble provides evidence that the Milky Way's SMBH occasionally becomes an active galactic nucleus (AGN). If, instead, the Fermi bubble was powered by supernovae, superbubbles, and other manifestations of massive-star and star-cluster formation and feedback, then the Milky Way's CMZ may be analogous to nearby starbursts such as M82 and may power a weak nuclear superwind. It is possible that both star formation and the SMBH contribute momentum, energy, and mass to Fermi bubbles. The CMZ gas must play a central role in these processes. It either powers massive star formation and consequent massive-star driven feedback, or its behavior contributes the fuels for the occasional activity of the SMBH.

\section{The Herschel Galactic plane survey: Hi-GAL}

The space missions of the last decade have collected remarkable data probing the CMZ. The Fermi and Chandra (CXO) satellites mapped the distribution of $\gamma$-ray and X-ray emission from the CMZ and the high-latitude sky above the Galactic plane. The Spitzer, WISE, and AKARI missions have mapped the near- to far-infrared emission. The 3.5 meter Herschel Space Observatory mapped the entire Galactic plane over a $2^{\circ}$ Galactic latitude region in five far-IR to sub-mm bands. The development of ground-based bolometric focal plane arrays at sub-mm and $\mathrm{mm}$ wavelengths, single dish surveys, and interferometers such as the VLA and ALMA have also had a profound impact on studies of the CMZ.

The Hi-GAL survey (Molinari et al. 2010a, b; 2011) mapped 700 square degrees (the open-time key project Hi-GAL and its successor programs, Hi-GAL360, and $2 \pi$-GAL received over 900 hours of observing time on Herschel, making it the largest program 


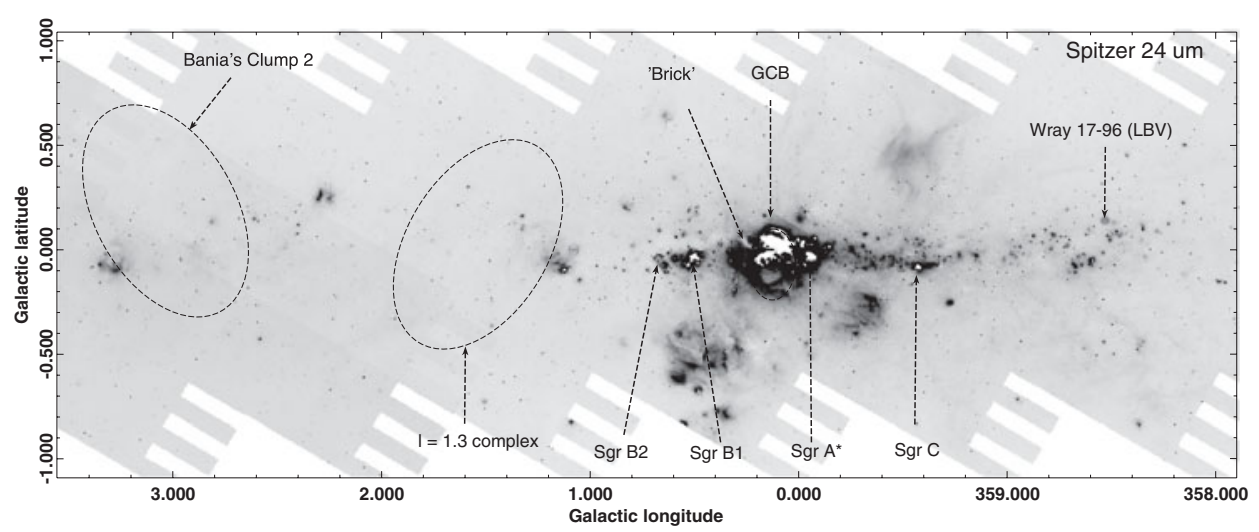

Figure 2. The central molecular zone at $24 \mu \mathrm{m}$ as observed with Spitzer with several of the major regions marked. The Galactic center bubble (GCB) located between Sgr A* and the dark cloud G0.25+0.02 (the 'Brick') is saturated at $24 \mu \mathrm{m}$. Wray 17-96 is a dust shell surrounding an LBV; it is the most prominent post-main-sequence object in this image.

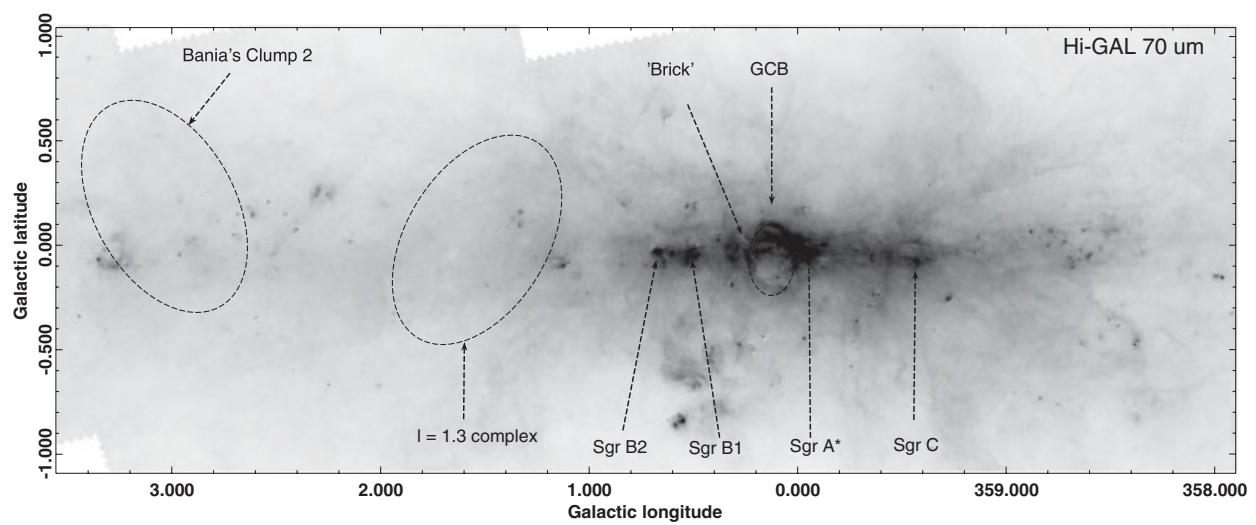

Figure 3. The central molecular zone at $70 \mu \mathrm{m}$ as observed with Herschel. Note that the warmest dust extends from Sgr C to about Sgr B2, which are separated by approximately 200 pc in projection.

executed on Herschel) in five broad-band filters; 70, 160, 250, 350, and $500 \mu \mathrm{m}$. The HiGAL team is led by Sergio Molinari in Rome and has approximately 150 co-investigators.

Figure 2 through 4 show the CMZ from $l=358^{\circ}$ to $l=3.6^{\circ}$ as imaged with Spitzer and Herschel between 24 and $350 \mu \mathrm{m}$. While the shorter wavelengths at 24 and $70 \mu \mathrm{m}$ trace warm dust associated with HII regions, cloud edges heated by external UV radiation fields, massive protostars, and the envelopes of post main-sequence objects, the longer wavelength Hi-GAL channels trace the cool dust associated with GMCs. Figures 2, 3, and 4 illustrate the CMZ between 24 and $500 \mu \mathrm{m}$.

Most star formation in the CMZ occurs within 100 pc of Sgr A*. This region contains at least three massive clusters; the nuclear star cluster surrounding Sgr A*, the massive but compact Arches cluster, and the somewhat dispersed Quintuplet cluster. The most prominent feature in the 24 and $70 \mu \mathrm{m}$ images is the Galactic center bubble (GCB). This feature is about $800^{\prime \prime}$ (33 pc) by $1200^{\prime \prime}$ (50 pc) in diameter and surrounds the Arches and Quintuplet clusters. The high latitude rim of the GCB consists of the ionized 'Arched Filaments' presumably heated and ionized by massive OB stars in and around the Arches 


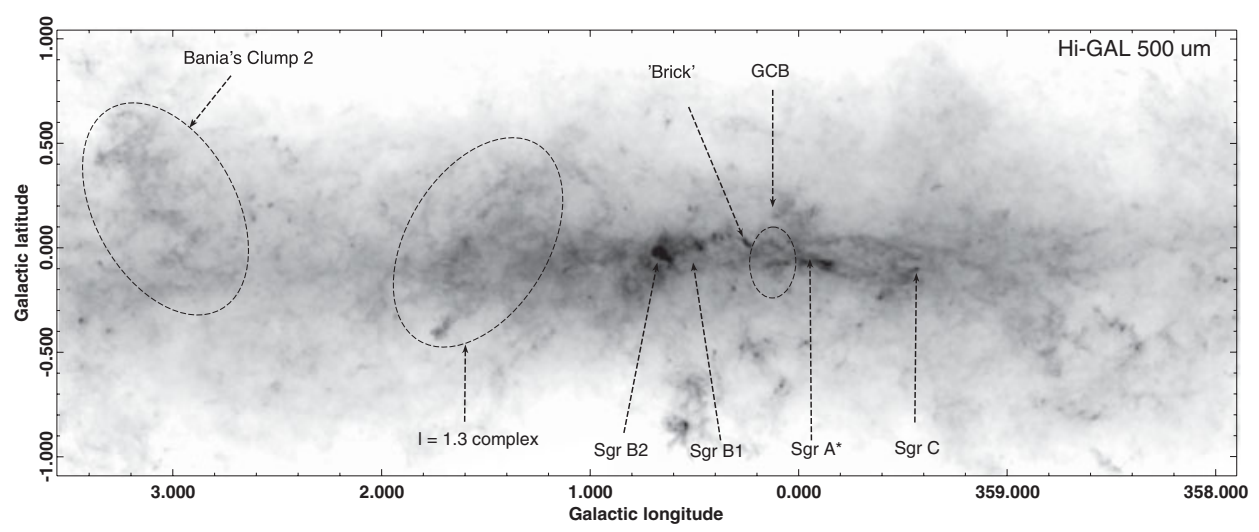

Figure 4. The central molecular zone at $500 \mu \mathrm{m}$ as observed with Herschel. Comparison with Figure 2 shows that while two thirds of the cool dust traced by $500 \mu \mathrm{m}$ is located at positive Galactic longitudes, most of the compact $24 \mu \mathrm{m}$ source are located at negative longitudes.

cluster. The high-longitude rim of the GCB coincides with the brightest groups of nonthermal radio filaments in the CMZ that cross the Galactic plane around longitude $0.18^{\circ}$.

The most extreme infrared dark cloud in the Galaxy, G0.25+0.02 (a.k.a. the 'Brick') is located just outside the GCB in the dark dust lane that runs from the high longitude side of the GCB to Sgr B2 and beyond. This cloud (Longmore et al. 2012) has a mass of over $10^{5} \mathrm{M}_{\odot}$ and mean radius of about $2.5 \mathrm{pc}$. Is is completely opaque at $24 \mu \mathrm{m}$ and still seen in silhouette against the warm dust background at $70 \mu \mathrm{m}$. The Brick starts to emit at $160 \mu \mathrm{m}$, and is one of the brighter dust emission sources at wavelengths of $250 \mu \mathrm{m}$ and longer. Despite having an $\mathrm{H}_{2}$ density higher than $10^{4} \mathrm{~cm}^{-3}$, there is little evidence for on-going or recent star formation. Only one maser is associated with the Brick. Recent ALMA Cycle 0 observations show that this source is associated with a compact $3 \mathrm{~mm}$ dust clump. However, there are no HiI regions or any other signs of recent star formation in this massive and dense clump.

Figures 2 and 3 show that there are three other regions of recent massive star formation at longitudes higher than the Brick. About $5^{\prime}$ to $6^{\prime}$ to the lower-left, there is a diffuse concentration of 24 and $70 \mu \mathrm{m}$ dust emission which may trace a population of distributed massive stars around $[l, b]=[0.30,-0.05]$. Next is the distributed Sgr B1 complex around $[l, b]=[0.50,-0.07]$. The very young and luminous Sgr B2 star forming complex at $[l, b]=$ $[0.67,-0.05]$ is embedded in what may be the most massive and turbulent GMC in the entire Galaxy. Most of its embedded ultra-compact and compact HiI regions are obscured at $24 \mu \mathrm{m}$.

The $24 \mu \mathrm{m}$ image, and to some extent the $70 \mu \mathrm{m}$ image, shows a large concentration of compact emission sources exetending from Sgr A towards negative longitudes. However, the only currently active site of massive star formation is the $\operatorname{Sgr} \mathrm{C}$ complex $[l, b]=$ $[359.43,-0.10]$. Spectroscopy and the symmetric nature of the emission from some of these sources indicate that they are dusty, post-main-sequence objects with the most prominent one being the dusty ring nebula labeled as Wray 17-96, a spectroscopically confirmed luminous blue variable (LBV). Thought to lie in the foreground portion of the Galactic disk, its location among the cluster of compact $24 \mu \mathrm{m}$ sources between longitudes 358.2 and 0 suggests that a re-analysis of the distance may be warranted. The narrow-spread of the latitude distribution of the negative-longitude $24 \mu \mathrm{m}$ sources is similar to that of the dense CMZ gas, indicating that these objects are likely to be associated with the CMZ. 


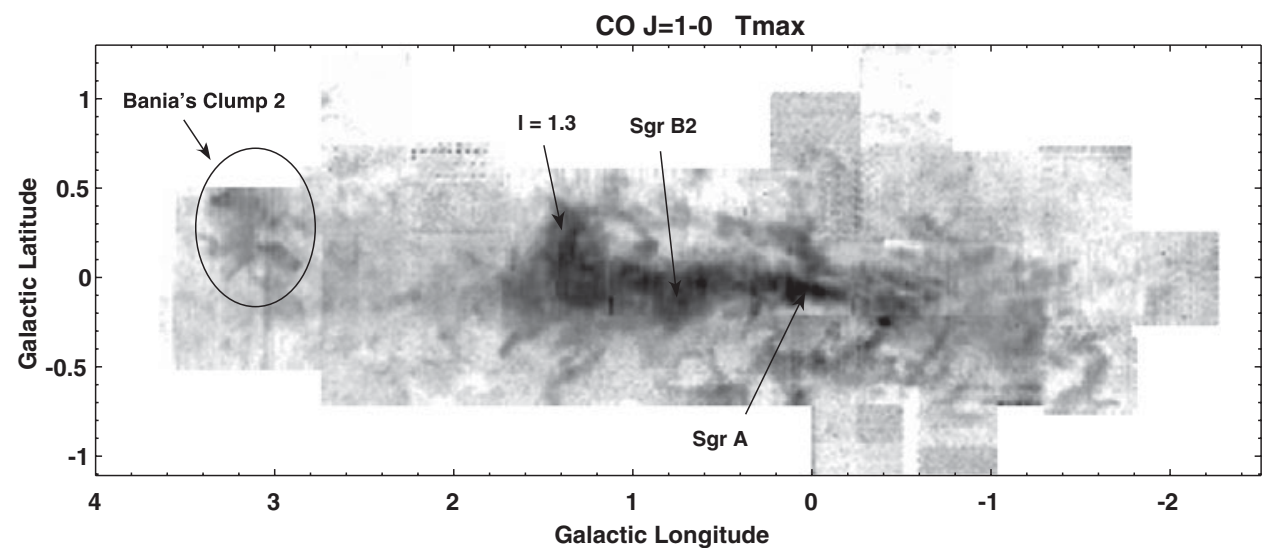

Figure 5. $115 \mathrm{GHz} \mathrm{CO} J=(1-0)$ peak intensity showing the CMZ and Bania's Clump 2. Taken from the Bally et al. (1987) AT\&T Bell Labs survey.

These maps also show prominent warm dust filaments above and below the plane that coincide with the ionized bubble found by Sofue and Handa (1984; see Law et al. 2009; 2010). A prominent,but diffuse dust streamer at $70 \mu \mathrm{m}$ extends from $[l, b]=[359.5,0.08]$ to about $[l, b]=[358.5,0.6]$. At positive longitudes the non-thermal radio filaments at $l=0.18^{\circ}$ connect to a dust ridge that can be traced to $[l, b]=[0.04,0.8]$; this structure has been noted for its braided double helix structure. It is associated with molecular gas traced by CO emission (Enokiya et al. 2014).

Three remarkable features of the CMZ stand out in the comparison of Figures 2, 3, and 4: First, over two-thirds of the cool dust is associated with the CMZ is located at positive longitudes. Second, most of the warm dust, especially dust associated with compact 24 $\mu \mathrm{m}$ sources are on the opposite side of Sgr A* at negative longitudes. Third, most star formation activity as indicated by either $24 \mu \mathrm{m}$ warm dust or $20 \mathrm{~cm}$ radio emission is confined to the inner part of the CMZ within $0.8^{\circ}(\sim 100 \mathrm{pc})$ of Sgr $\mathrm{A}^{*}$.

There are several diffuse sources of dust emission below the mid-plane in Figure 2. These objects are thought to be foreground HiI regions probably located in the Scutum arm (see discussion and references in Bally et al. 2010) at distances of order 3.5 to $4 \mathrm{kpc}$ from the Sun.

Moving to the outer CMZ, Figure 4 shows two massive concentrations of dust around $l=1.3^{\circ}$ and $3.5^{\circ}$, labeled as the ' $l=1.3$ complex' and 'Bania's Clump 2', respectively. Comparison with Figures 2 and 3 show that neither complex is actively forming stars since there are no prominent HII regions or compact 24 or $70 \mu \mathrm{m}$ sources in their directions. Figures 5 and 6 show the distribution of CO emission in the CMZ and the kinematics of Bania's Clump 2.

Bania's Clump 2 (Stark \& Bania 1986) has a remarkably large velocity spread. In a region smaller than $0.5^{\circ}(\sim 70 \mathrm{pc}$ in projection $)$, gas is spread over a greater than $150 \mathrm{~km} \mathrm{~s}^{-1}$ velocity range. This velocity extent is even extreme for the CMZ. As is typical of CMZ clouds, this object is bright in high-dipole moment molecules such as HCN and CS, indicating that it is dense. Three interpretations concerning the nature of Bania's Clump 2 have been put forth: (1) Clump 2 is a collection of clouds at the cusped-end of the innermost $x_{1}$ orbit where the orbits become self-intersecting. In this region, clouds approach apocenter and also start their plunge back towards pericenter. This model predicts both negative and positive LSR velocities and fails to explain why Clump 2 only exhibits positive radial velocities. (2) As suggested by Rodriguez-Fernandez et al. (2006), Clump 2 


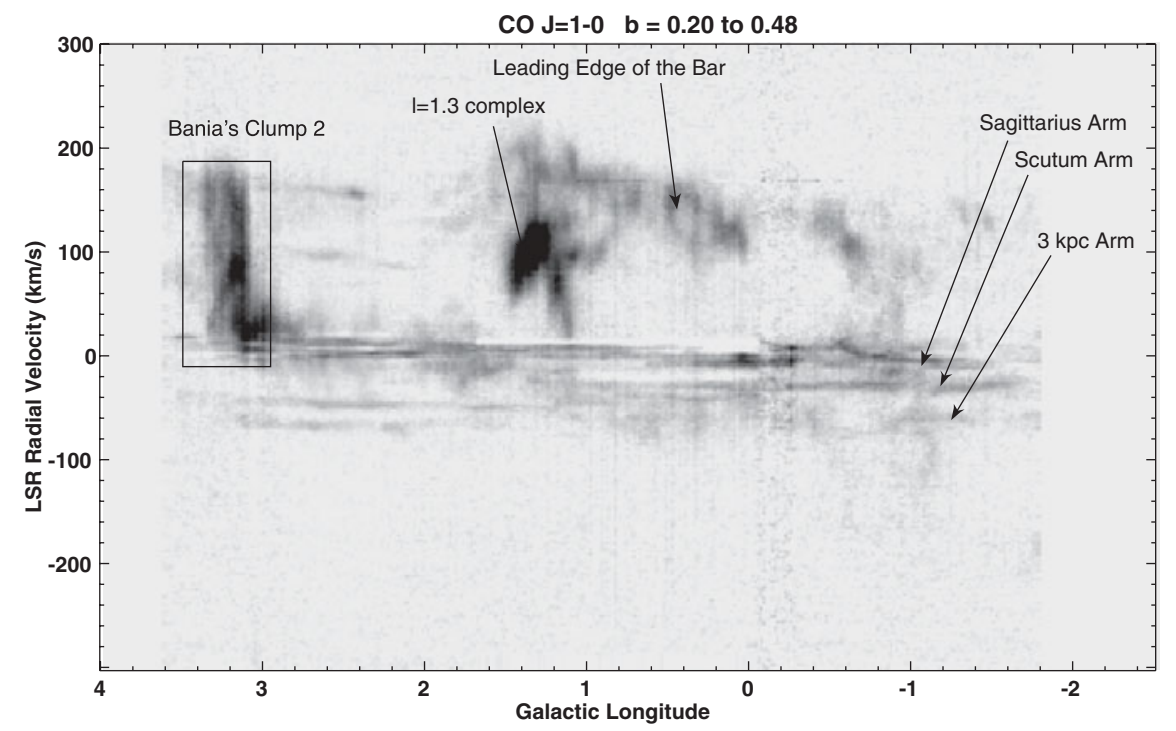

Figure 6. Spatial-velocity diagram showing the kinematics of all $\mathrm{CO}$ emitting gas between Galactic latitudes $0.20^{\circ}$ and $0.48^{\circ}$. Bania's Clump 2 extends over a velocity range of more than $150 \mathrm{~km} \mathrm{~s}^{-1}$. Most of the dense CMZ gas is missing from this plot because it is located below a latitude of $0.2^{\circ}$. Taken from the Bally et al. (1987) AT\&T Bell Labs survey.

may be located where the gas plunging in on an innermost $x_{1}$ orbit interacts with gas in the outer $x_{2}$ orbits. This model explains the large positive radial velocity, but might be expected to produce a two-valued velocity distribution with one value corresponding to the expected motion near pericenter, and an other associated with decelerated gas. This model does not naturally reproduce the nearly continuous velocity spread from $\mathrm{V}_{L S R} \sim 0$ to $180 \mathrm{~km} \mathrm{~s}^{-1}$. (3) Clump 2 may trace a nearly end-on dust lane along the leading edge of the bar associated with a inner $x_{1}$ orbit. The smooth emission as a function of radial velocity traces the acceleration of gas as it falls from apocenter towards pericenter in the gravitational potential of the central bulge and bar. This model explains in a natural way why all Clump 2 gas is at positive LSR velocities. However, it requires that our line-of-sight is nearly parallel to the leading dust lane in the Galactic bar.

\section{Dust temperature and column density maps: a 100 pc ring?}

The availability of data throughout the infrared and sub-millimeter wavelength ranges makes it possible to estimate the beam-averaged line-of-sight dust temperatures and column densities. Battersby et al. (2011) used the Herschel data to estimate these parameters on a pixel-to-pixel basis. Their approach is to iteratively separate the smooth 'cirrus-like' background thought to be associated with the low-density components of the ISM from the dust associated with dense gas. Once the dense and diffuse components are separated, an interpolated smooth version of the background is subtracted from each pixel associated with the dense component as an approximate correction for unrelated foreground and background dust emission. The resulting spectral energy distributions at each pixel are used to estimate dust temperatures. The measured fluxes are then converted to estimates of the dust and associated gas column densities at each pixel using the dust opacities appropriate for each frequency and an assumed gas to dust ratio (see Battersby 


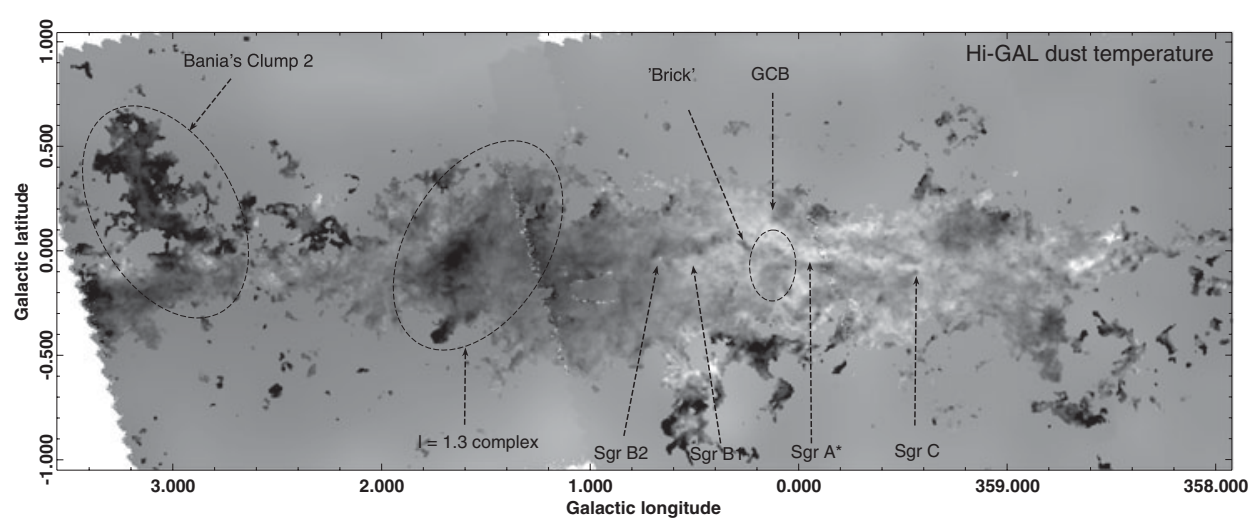

Figure 7. Dust temperature map of the CMZ using the Battersby (2011) algorithm which iteratively separates the smooth (background) emission from the more structured emission thought to arise from dense clumps.

et al. 2011 for the details). The resulting dust temperature and column density maps are shown in Figures 5 and 6.

Inspection of these images shows that warm dust is widespread between longitudes of $l=359.4^{\circ}$ (the location of Sgr C) and $0.8^{\circ}$ (the location of Sgr B2). The line-of-sight averaged dust temperatures range from 30 to $50 \mathrm{~K}$ in regions between the dense CMZ clouds such as the Brick. The warm dust distribution also extends to negative longitudes around $l=358.5^{\circ}$ where most of the $24 \mu \mathrm{m}$ sources are located. However, at positive longitudes beyond $l=0.8^{\circ}$ and in the compact clumps such as the Brick and the 20 $\mathrm{km} \mathrm{s}^{-1}$ cloud near Sgr A*, the dust emission is cold with temperatures ranging from 15 to $25 \mathrm{~K}$ The coldest dust is seen in the $l=1.3^{\circ}$ complex and in Bania's Clump 2 .

Comparison with Figure 6 shows that warm dust and high column densities are anticorrelated. The highest column density dust clouds are located between $l=359.4^{\circ}(\mathrm{Sgr} \mathrm{C})$ and $0.8^{\circ} \mathrm{Sgr} \mathrm{B2}$ ). For an assumed gas-to-dust ratio of 100 , the beam-averaged peak column density (in a 35" beam) in Figure 6 is $N\left(H_{2}\right)=2 \times 10^{24} \mathrm{~cm}^{-2}$; this value is reached only in Sgr B2. The total mass of this cloud is $\sim 10^{6} \mathrm{M}_{\odot}$. The Brick is also one of the highest column density clouds in the CMZ with $N\left(H_{2}\right)=2.5 \times 10^{23} \mathrm{~cm}^{-2}$ and a total mass of about $10^{5} \mathrm{M}_{\odot}$.

The cold, high column dust forms an 'infinity sign' shaped distribution which forms a ring with a mean Galactocentric radius of about 100 pc (Molinari et al. 2011). The shape of this dust ring suggests that the vertical oscillation frequency in the Galactic center gravitational potential of test particles is a factor of two greater than the orbital frequency. Molinari et al. (2011) use published spectra to determine the radial velocities of the ring and conclude that it is asymmetrically placed with respect to Sgr A*. In their interpretation, $\operatorname{Sgr} \mathrm{C}$ should lie near the approaching tangent point of the ring while Sgr B2 should lie at the receding tangent point. Their low radial velocities $\left(\mathrm{V}_{L S R} \approx-60\right.$ and $+65 \mathrm{~km} \mathrm{~s}^{-1}$, respectively) are interpreted as an indication that the ring is highly elongated with its major axis lying nearly perpendicular to our line-of-sight. In this picture, both Sgr C and B2 lie near the orbit's apocenter.

The orbital motions of clouds and stars indicates that the equivalent circular velocity about the Galactic center potential varies from about $120 \mathrm{~km} \mathrm{~s}^{-1}$ within a few parsecs of Sgr A* (but well outside the SMBHs gravitational influence), to about 140 to $180 \mathrm{~km} \mathrm{~s}^{-1}$ at a Galactocentric radius of a few hundred pc. The slight rise in the equivalent circular velocity can be interpreted as indicative of a density distribution which, in the point 


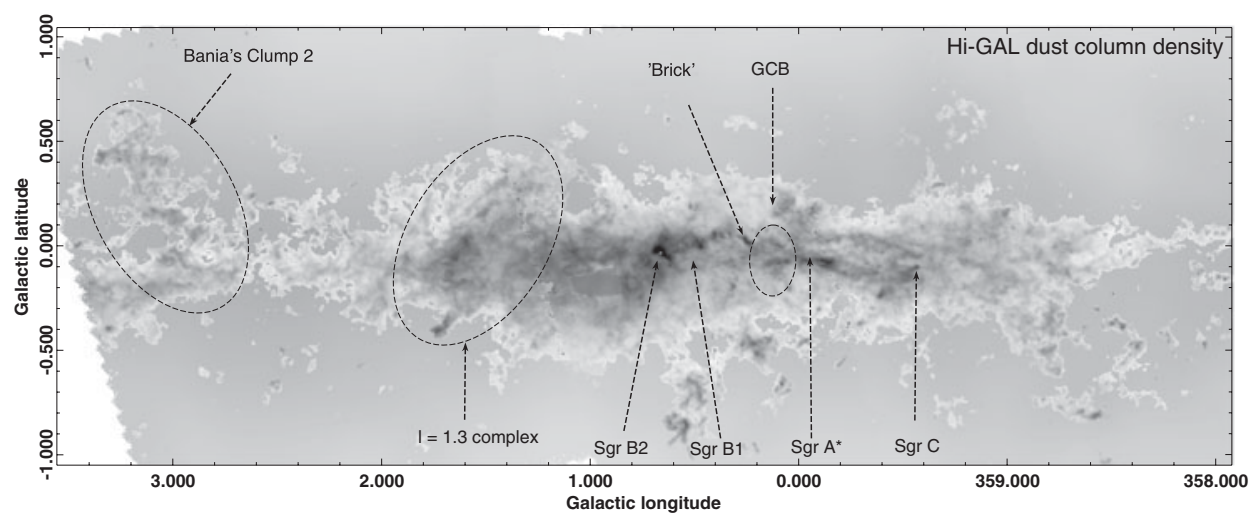

Figure 8. Dust column density map of the CMZ using the Battersby (2011) algorithm which iteratively separates the smooth (background) emission from the more structured emission thought to arise from dense clumps.

symmetric approximation, declines as $r^{-1.8}$. In such a potential, a test particle on an eccentric orbit will move faster than the equivalent circular velocity at pericenter, and slower than that reference speed at apocenter.

Such a ring had also been postulated by Rodriguez-Fernandez et al. (2006). However, the ring proposed by these authors extends well beyond Sgr B2 to at least $l=1.2^{\circ}$, a bit beyond the Sgr D complex of HiI regions. The ratio of proper motions to radial velocities could in principle distinguish the Molinari 100 pc radius ring from the larger RodriguezFernandez version. In the former model, the proper motion of Sgr B2 and Sgr C should be near $0 \mathrm{~km} \mathrm{~s}^{-1}$ while in the larger ring model, there should be measurable proper motion. VLBA measurement of the motions of maser spots in Sgr B2 with respect to Sgr A* indicate that Sgr B2 may be moving towards positive longitudes with a velocity of order $80 \mathrm{~km} \mathrm{~s}^{-1}$ (Reid et al. 2008). Thus, if these motions are a true indication of the overall motion of Sgr B2, then it can not be near the tangent point, and the CMZ's starburst ring must have a larger apocenter. Unfortunately, no proper motion measurements exist for Sgr C.

\section{ALMA observations: the Brick}

As discussed above, the extreme IRDC G0.25+0.02 (a.k.a. the 'Brick' - because it is optically thick as one), is remarkable for its small size, high column density, volume density, and apparent lack of star formation. As is typical for CMZ molecular clouds, the line widths are large. Figure 9 shows a channel map from a recent ALMA observation of the $3 \mathrm{~mm}$ lines such as $\mathrm{HCO}^{+}$. Comparison of the intensities with $\mathrm{H}^{13} \mathrm{CO}^{+}$indicate that the $J=(1-0)$ line is optically thick with $\tau \sim 2$ to 5 . The blue-shifted side of the Brick $\mathrm{HCO}^{+}$and $\mathrm{HCN}$ emission is laced with narrow (less than $2^{\prime \prime}$ wide) filaments of absorbing gas seen in silhouette against the brighter, optically thick emission. Though most of these narrow absorption filaments have line widths less than $10 \mathrm{~km} \mathrm{~s}^{-1}$, one set of broad-line absorption filaments are seen with a line width larger than $20 \mathrm{~km} \mathrm{~s}^{-1}$ (the long filament running diagonally across the figure near the top). This structure is less than $2^{\prime \prime}(0.04-0.08 \mathrm{pc})$ wide and more than $50^{\prime \prime}$ long and has an optical depth of about 0.5. The $\mathrm{HCO}^{+}$emission has a peak brightness of only 5 to $10 \mathrm{~K}$ with a mean value of only 2 to $4 \mathrm{~K}$. It appears to be area filling. Estimates of gas temperatures based on $\mathrm{NH}_{3}$ or $\mathrm{H}_{2} \mathrm{CO}$ emission indicate that the $\mathrm{CMZ}$ gas is warm with $\mathrm{T} \sim 60$ to $100 \mathrm{~K}$. The low 


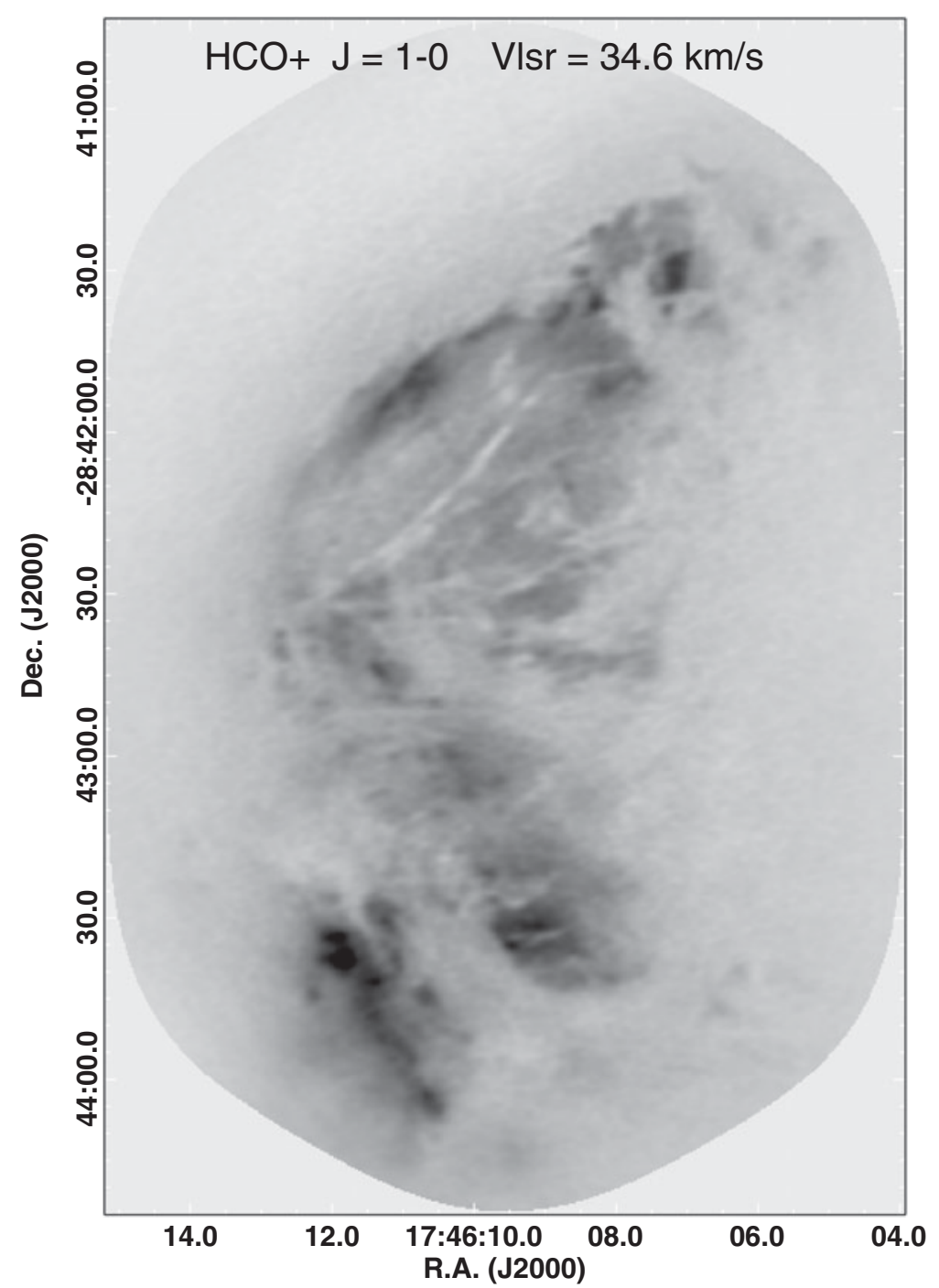

Figure 9. The 2.5 pc cloud G0.25+0.02 (a.k.a. the 'Brick', which is the most extreme IRDC in the Galaxy) showing one channel from $\mathrm{HCO}^{+} J=(1-0)$ data cube from Cycle-0 ALMA observations. The cloud is shown in R. A.-Dec. coordinates with North towards the top and East towards the left. The cloud emission spans a radial velocity range of over $40 \mathrm{~km} \mathrm{~s}^{-1}$ and exhibits a large velocity gradient opposite to the sense of Galactic rotation. Comparison of the $\mathrm{H}^{12} \mathrm{CO}^{+}$ with the $\mathrm{H}^{13} \mathrm{CO}^{+}$intensities shows that the $\mathrm{H}^{12} \mathrm{CO}^{+}$emission is optically thick with $\tau \sim 2$ to 5 . Note the long filaments seen in absorption. The upper part of the Brick, lying at larger Galactic longitudes has a lower radial velocity than the bottom part located at lower longitudes. Taken from a paper in preparation presenting the ALMA data. See the contributions by Steve Longmore and Jill Rathborne for more details.

peak brightness temperatures of the optically thick $3 \mathrm{~mm}$ lines may be an indication that the excitation of $\mathrm{HCN}$ and $\mathrm{HCO}^{+}$is sub-thermal at and in front of the $\tau \approx 1$ surface. The absorption filaments must have even lower excitation temperatures. 
If this broad-line absorption feature is as deep along the line-of-sight as it is wide, then its optical depth places a severe constraint on the abundance of $\mathrm{HCO}^{+}$. If the physical depth of this feature is $3 \times 10^{17} \mathrm{~cm}$, and the excitation is highly sub-thermal as indicated by the low-level of emission ( $\operatorname{say~} \mathrm{n}\left(\mathrm{H}_{2}\right)<10^{3} \mathrm{~cm}^{-3}$ ), but the optical depth is near unity, then the relative abundance of $\mathrm{HCO}^{+}$relative to $\mathrm{H}_{2}$ must be at least $\sim 10^{-7}$.

These preliminary ALMA results show that this remarkable facility will revolutionize studies of the CMZ.

\section{What powers the Sofue-Handa lobe and Fermi/LAT bubbles?}

As discussed in the Introduction, the Fermi/LAT bubbles and associated phenomena indicate the presence of a bipolar flow emerging from the central regions of the Galaxy with sufficient momentum to reach heights of 5 or $6 \mathrm{kpc}$ above and below the Galactic plane. Is this bubble powered by occasional outbursts of the Galaxy's SMBH? Or, is it powered by star formation activity and its consequences?

The star formation rate (SFR) in the CMZ has been estimated from counts of infrared sources, massive stars, radio free-free emission, masers, and HiI regions and is found to be between 0.02 and $0.1 \mathrm{M}_{\odot} \mathrm{yr}^{-1}$ (Morris \& Serabyn 1996; Yusef-Zadeh, et al. 2009; Longmore et al. 2013a). Dividing the current mass $\left(3-7 \times 10^{7} \mathrm{M}_{\odot}\right)$ of the CMZ by the SFR gives a dense-gas depletion time of order $\tau_{\text {deplete }}=3 \times 10^{8}$ to $5 \times 10^{9}$ years. Assuming that the inner bulge is made entirely of stars, has a stellar mass $\sim 5 \times 10^{9} \mathrm{M}_{\odot}$ (estimated from a circular velocity of $200 \mathrm{~km} \mathrm{~s}^{-1}$ at a radius of $500 \mathrm{pc}$ ), that it formed from CMZ clouds, its formation would require an average star formation rate of $\sim 0.5$ $\mathrm{M}_{\odot} \mathrm{yr}^{-1}$. The star formation rate in the distant past may have been much larger, or the bulge may have been built by the merging of smaller galaxies with the Milky Way.

The star formation rate per unit mass of dense $\left(\mathrm{n}\left(\mathrm{H}_{2}\right)>10^{4} \mathrm{~cm}^{-3}\right)$ gas may be lower in the Galactic center than in the Galactic disk. While $80 \%$ of the $1.3 \mathrm{~cm} \mathrm{NH}$ emission (a tracer of dense gas) in the Milky Way's Galactic plane is in the CMZ, less than $5 \%$ of the Milky Way's stars form there (Longmore et al. 2013a). Kruijssen et al. (2013) explored possible reasons for a depressed star formation rate per unit mass of dense gas. They considered turbulence, tidal stresses caused by the motion of clouds along eccentric orbits in the CMZ potential, tidal shocking, strong magnetic support, cloud-cloud collisions, and other effects.

The continued presence of a CMZ indicates that gas and dust is accreted into this region at a rate which approximately balances the SFR plus the expulsion of gas by either massive-star feedback or occasional AGN activity. The most likely source of replenishment is the orbit decay of clouds in the Galactic disk driven by its interaction with the central stellar bar. Elmegreen (1994) suggested that the CMZ gas may experience quasi-periodic episodes of enhanced star formation (starbursts), interspersed by periods of low star formation rate. Such 'relaxation-oscillations' may be powered by the feedback from massive stars.

Eccentric orbits bring dense clouds moving in the $100 \mathrm{pc}$ or larger ring on the innermost $x_{2}$ orbits to with 20 to $40 \mathrm{pc}$ of the nucleus (Molinari et al. 2011). Tidal shearing and compression by the deep gravitational potential well of the central bulge stretches and compresses these clouds as they pass through pericenter. The stretching can cause the portions of clouds closer to the center to overtake the outer parts, resulting in reverse velocity gradients as observed in the Brick. Compression along the latitude direction can trigger gravitational collapse and star formation. Kruijssen et al. (2013) suggest that massive clouds can experience a burst of star formation within 0.5 to $2 \mathrm{Myr}$ of pericenter passage. In this scenario, the $20 \mathrm{~km} \mathrm{~s}^{-1}$ cloud is about to experience pericenter 
passage and is getting sheared along the Galactic plane. The Brick passed pericenter about 170,000 years ago. Sgr B2, passed through pericenter about a Myr ago (the time depends on its poorly constrained proper motion) and is now in the middle of a major burst of star formation. Sgr B1, assumed to be on the far-side and slightly below the 100 pc ring, is now blowing its parent cloud apart. By the time these complexes move behind the Galactic center to the far side of the ring and orbit to negative longitudes about 2 to $5 \mathrm{Myr}$ after pericenter passage, ionization and the explosions of the most massive stars disrupt their parent clouds. The asymmetric distributions of the dense gas and $24 \mu \mathrm{m}$ sources may provide support for such a model.

However, a key question is: Do the $24 \mu \mathrm{m}$ sources truly trace the distribution of massive stars through the warm dust associated with their HiI regions, or is the opacity of the CMZ dense cloud sufficient to hide a large population of highly embedded massive protostars? ALMA and VLA observations in the near future may either identify such an embedded population, or may show that such a population does not exist. Either way, the presence of a large number of $24 \mu \mathrm{m}$ sources at negative longitudes where only a small fraction of the gas is located suggest that instead of tracing a very young $(<1 \mathrm{Myr}$ old) population of massive young stars, they instead trace a somewhat older generation of massive stars formed perhaps a few to 20 Myr ago.

The presence of hundreds of massive young stars in the CMZ and current star formation rates of order $0.1 \mathrm{M}_{\odot} \mathrm{yr}^{-1}$ imply that feedback from massive stars by means of ionization and supernovae can generate sufficient momentum and energy to occasionally completely disrupt parts of the CMZ. The current sources of energy input may provide a clue. The GCB appears to be energized by massive star feedback from the Arches and Quintuplet clusters while Sgr $\mathrm{A}^{*}$ and the central cluster lie outside the GCB boundaries. The mass in the GCB walls is about $10^{4}$ to $10^{5} \mathrm{M}_{\odot}$, and the structure is expanding with a velocity of order $50 \mathrm{~km} \mathrm{~s}^{-1}$, implying a kinetic energy of order 0.25 to $2.5 \times 10^{51} \mathrm{ergs}$. At least several to several dozen $\mathrm{SNe}$ are required to produce this much kinetic energy since much of the initial blast-wave kinetic energy is radiated away. The energetics of the Sofue-Handa lobe and Fermi/LAT bubble are much less certain since the observations only trace minor components.

The Milky Way's total SFR is around 1 to $3 \mathrm{M}_{\odot} \mathrm{yr}^{-1}$ (Murray \& Rahman 2010). Its supernova $(\mathrm{SN})$ rate is about 1 per 100 years on average, half of which are probably Type II core-collapse SNe. The SN rate should be similar to the birth-rate of stars more massive than $8 \mathrm{M}_{\odot}$, the least massive to explode. Scaling these numbers to the CMZ, a star formation rate of $0.1 \mathrm{M}_{\odot} \mathrm{yr}^{-1}$ would produce one Type II SN every 2,000 to 6,000 years on average. If star formation proceeds at this rate for more than a few Myr (say $10 \mathrm{Myr}), 5-15 \times 10^{3} \mathrm{SNe}$ would eventually explode, injecting about $0.5-1.5 \times 10^{55}$ ergs into the CMZ environment (assuming each SN injects $10^{51}$ ergs of kinetic energy). Such an injection of energy can dissociate and disrupt the CMZ clouds. While clouds at longitudes higher than $0.8^{\circ}$ lack significant current star formation, the large population of $24 \mu \mathrm{m}$ sources at negative longitudes may be remnants of a starburst in the CMZ about 5 to $20 \mathrm{Myr}$ ago. The $10^{3}$ to $10^{4} \mathrm{SNe}$ may have disrupted this portion of the CMZ to produce the CMZ gas/dust and $24 \mu \mathrm{m}$ asymmetries. For a normal IMF, an SFE of $10 \%$ in $10^{7} \mathrm{M}_{\odot}$ of progenitor gas could produce $10^{4} \mathrm{SNe} .10^{4} \mathrm{SNe}$ could dissociate and accelerate $10^{7} \mathrm{M}_{\odot}$ to velocities of $\sim 400 \mathrm{~km} \mathrm{~s}^{-1}$. The remnant gas could reach many kpc above and below the plane. 


\section{Conclusions}

Space missions such as Spitzer, Herschel, WISE, AKARI, the SOFIA aircraft, and ground based facilities such as Mopra, APEX, GBT, VLA, VLBA, and ALMA are poised to revolutionize our understanding of the $\mathrm{CMZ}$ and the processes occurring there. Herschel Hi-GAL has provided the best 60 to $500 \mu \mathrm{m}$ broad-band dust continuum data that we are likely to see in our lifetime. It will be mined for decades. However, the analysis of existing and soon to be gotten data will challenge us. We are likely to me more limited by brain-power than computer power in our attempts to understand the complex ecology of the CMZ.

We now know that we live in a mildly barred galaxy with a massive CMZ. The CMZ clouds are orders of magnitude denser and more turbulent than GMCs in the Galactic disk. Extensive star formation capable of producing the most massive star clusters and $\mathrm{OB}$ associations in the Galaxy are confined to the central $100 \mathrm{pc}$ radius region surrounding the SMBH. The outer parts of the CMZ, despite containing vast reservoirs of dense gas, are relatively inactive star formers. The rate of star formation may be variable. But the rate of recent star formation may be sufficient to disrupt large portions of the CMZ and to power the nested set of bubbles seen to emerge from the CMZ towards high Galactic latitudes.

Many important questions remain unanswered. Why are the distributions of dense gas and dust so asymmetric? Why do the $24 \mu \mathrm{m}$ sources show the opposite symmetry as the CMZ gas and dust? Is this an artifact of extinction, or a measure of the true distribution of massive stars? What is the interplay between CMZ star formation and the SMBH? Does the SMBH have any role in regulating CMZ star formation? Or does star formation regulate the growth rate and feeding of the SMBH? Can the 'X-ray light echoes' produced by the occasional outbursts of the $\mathrm{SMBH}$ be used to constrain the $3 \mathrm{D}$ structure of the CMZ gas? How does star formation work in the central parsecs? What is the average star formation rate in the CMZ? Is the dependence of this rate on the gas surface and volume densities the same as found in the Galactic disk and in nearby galaxies? What can we infer about star formation and SMBH evolution in the high pressure environments of distant starburst and sub-mm galaxies?

The future is exciting with phenomenal new tools becoming available to address these problems. But, the human resources to take advantage of these tools must also be nurtured in order to make progress.

\section{References}

Bally, J., Aguirre, J., Battersby, C., et al. 2010, ApJ 721, 137

Bally, J., Stark, A. A., Wilson, R. W., \& Henkel, C. 1988, ApJ 324, 223

Bally, J., Stark, A. A., Wilson, R. W., \& Henkel, C. 1987, ApJS 65, 13

Battersby, C., Bally, J., Ginsburg, A., et al. 2011, A\&A 535, A128

Binney, J., Gerhard, O. E., Stark, A. A., Bally, J., \& Uchida, K. I. 1991, MNRAS 252, 210

Blitz, L., Binney, J., Lo, K. Y., Bally, J., \& Ho, P. T. P. 1993, Nature 361, 417

Carretti, E., Crocker, R. M., Staveley-Smith, L., et al. 2013, Nature, 493, 66

Clavel, M., Terrier, R., Goldwurm, A., et al. 2013, A\&A 558, A32

Elmegreen, B. G. 1994, ApJ 425, L73

Enokiya, R., Torii, K., Schultheis, M., et al. 2014, ApJ 780, 72

Ferrière, K., Gillard, W., \& Jean, P. 2007, A\&SA 467, 611

Kormendy, J. \& Kennicutt, R. C., Jr. 2004, ARAA 42, 603

Kruijssen, J. M. D., Longmore, S. N., Elmegreen, B. G., et al. 2013, arXiv: 1303.6286

Law, C. J. 2010, ApJ 708, 474

Law, C. J., Backer, D., Yusef-Zadeh, F., \& Maddalena, R. 2009, ApJ 695, 1070 
Longmore, S. N., Kruijssen, J. M. D., Bally, J., et al. 2013a, MNRAS 433, L15

Longmore, S. N., Bally, J., Testi, L., et al. 2013b, MNRAS 429, 987

Longmore, S. N., Rathborne, J., Bastian, N., et al. 2012, ApJ 746, 117

Molinari, S., Bally, J., Noriega-Crespo, A., et al. 2011, ApJ 735, L33

Molinari, S., Swinyard, B., Bally, J., et al. 2010a, A\&A 518, L100

Molinari, S., Swinyard, B., Bally, J., et al. 2010b, PASP 122, 314

Morris, M. \& Serabyn, E. 1996, ARAA 34, 645

Murray, N. \& Rahman, M. 2010, ApJ 709, 424

Oka, T., Hasegawa, T., Sato, F., Tsuboi, M., \& Miyazaki, A. 1998, ApJS 118, 455

Oka, T., Hasegawa, T., Sato, F., et al. 2001, ApJ 562, 348

Planck Collaboration, Ade, P. A. R.. Aghanim, N., et al. 2013, A\& A 554, A139

Reid, M. J., Menten, K. M., Zheng, X. W., Brunthaler, A., \& Xu, Y. 2009, ApJ 705, 1548

Rodriguez-Fernandez, N. J., Combes, F., Martin-Pintado, J., Wilson, T. L., \& Apponi, A. 2006, A\&SA 455, 963

Sofue, Y. \& Handa, T. 1984, Nature, 310, 568

Stark, A. A. \& Bania, T. M. 1986, ApJ 306, L17

Su, M., Slatyer, T. R., \& Finkbeiner, D. P. 2010, ApJ 724, 1044

Tsuboi, M., Handa, T., \& Ukita, N. 1999, ApJS 120, 1

Yusef-Zadeh, F., Hewitt, J. W., Arendt, R. G., et al. 2009, ApJ 702, 178

Yusef-Zadeh, F., Braatz, J., Wardle, M., \& Roberts, D. 2008, ApJ 683, L147 